\title{
How far have we achieved by systemic therapy for recurrent/ metastatic nasopharyngeal carcinoma?
}

\author{
Victor Lee \\ Department of Clinical Oncology, Li Ka Shing Faculty of Medicine, The University of Hong Kong, Hong Kong, China \\ Correspondence to: Victor Lee. Department of Clinical Oncology, Queen Mary Hospital, 102 Pokfulam Road, Hong Kong, China. \\ Email: vhflee@hku.hk. \\ Provenance: This is an invited Commentary commissioned by Section Editor San-Gang Wu (Department of Radiation Oncology, Xiamen Cancer \\ Center, the First affiliated Hospital of Xiamen University, Xiamen, China). \\ Comment on: Zhang L, Huang Y, Hong S, et al. Gemcitabine plus cisplatin versus fluorouracil plus cisplatin in recurrent or metastatic nasopharyngeal \\ carcinoma: a multicentre, randomised, open-label, phase 3 trial. Lancet 2016;388:1883-92.
}

Submitted Oct 31, 2016. Accepted for publication Nov 08, 2016.

doi: $10.21037 /$ tcr.2016.12.25

View this article at: http://dx.doi.org/10.21037/tcr.2016.12.25

Nasopharyngeal carcinoma of undifferentiated type is distinctive in terms of radical and geographical distribution, with highest incidence in Southern China, Taiwan and Singapore, followed by North Africa and Alaska. Radiotherapy with contemporary techniques like intensitymodulated radiation therapy is the mainstay of treatment for early-stage disease, while concurrent chemoradiation with or without adjunct chemotherapy is indicated in locoregionally advanced disease (1). Nevertheless, about $30 \%$ of patients develop recurrence or metastasis despite intensive radical treatment (2). Though most of these relapsed patients have an unfavorable survival outcome, their survival can be significantly prolonged with palliative chemotherapy.

Traditionally platinum doublets have been the standard first-line chemotherapy. Among various different regimens, cisplatin and 5-FU is often the regimen of choice because of ample experience from oncologists as well as its favorable treatment response and tolerability. This popular regimen produced an overall response (OR) rate between $66 \%$ and $78 \%$ and a median survival of 12 to 14 months (3-5). In particular, Chi et al. revealed that all patients with locally recurrent disease responded to cisplatin, 5-FU and leucovorin producing a median survival of 34 months, while $80 \%$ of those with metastatic disease responded with a median survival of 14 months (5). This regimen was found effective even in patients who had received prior chemotherapy. All five patients who had earlier received mitoxantrone still responded, suggesting a lack of cross resistance. Another four patients with good responses to prior induction chemotherapy with cisplatin and 5-FU followed by radiotherapy who then developed metastatic disease with disease-free intervals greater than 1 year still, nevertheless, responded to the same regimen (5). Moreover, the toxicity profile is generally favorable with mild immunosuppression and peripheral neuropathy. However cisplatin-induced nephrotoxicity and ototoxicity were of concern, especially in patients who had also received cisplatin during their previous definitive chemoradiation. Not uncommonly, carboplatin has been used as a substitute of cisplatin for advanced head and neck cancers including NPC (6). Two older randomized controlled studies on advanced head and neck cancers demonstrated that cisplatin was superior to carboplatin in terms of improved response rate $(7,8)$. One of these showed cisplatin conferred superior disease-free survival and overall survival (OS) compared to carboplatin when both were used with 5-FU (8). However this study did not recruit patients with recurrent or metastatic diseases. In 2013, a retrospective Malaysian study compared cisplatin and 5-FU with carboplatin and $5-\mathrm{FU}$ in 41 patients with recurrent and metastatic head and neck squamous cell cancer and NPC (9). This showed that carboplatin and 5-FU (median survival, 12 months) was not inferior to cisplatin and 5-FU (median survival, 10 months; $\mathrm{P}=0.110$ ). However, drawbacks of this study were that no subgroup analysis was performed for NPC patients only and 
there were six treatment-related mortalities (14.6\%)-four in the carboplatin +5 -FU group and two in the cisplatin + 5-FU group. All these phase II retrospective and prospective trials are not convincing enough to routinely recommend $\mathrm{PF}$ as a standard regimen in the first-line setting and thus a phase III head-to-head randomized-controlled trial is definitely indicated to answer this question.

Zhang and colleagues were congratulated for their pivotal phase III trial using gemcitabine and cisplatin (GP) as first-line treatment for recurrent or metastatic NPC (10). This is the first multi-center randomized-controlled trial investigating the replacement of $5-\mathrm{FU}$ with gemcitabine in addition to cisplatin as $1^{\text {st }}$ line treatment in this setting, performed in endemic areas of NPC in 22 hospitals in China. The primary study endpoint was progression-free survival (PFS) in the intention-to-treat population, while secondary endpoints were objective response rate, disease control rate, safety profiles and OS. Altogether 362 patients were equally randomized to either GP or cisplatin with 5-FU (PF) regimen. After a median follow-up of 19.4 months, the median PFS was statistically longer in the GP regimen (median: 7.0 months) as compared to PF counterpart (5.6 months, $\mathrm{P}<0.0001)$. Subgroup analysis demonstrated that PFS advantage was observed in all prespecified subgroups except non-type-III histology and chemotherapy with 5 cycles. A higher objective response rate was noted in the GP regimen (64\%) compared to PF regimen (42\%), while disease control was similar between the two arms (90\% versus $86 \%$ ). With respect to safety profiles, more grade $\geq 3$ hematological adverse events were seen in patients treated with GP, whereas more mucosal inflammation was reported in those who received PF. Finally, OS was also significantly longer with GP compared to PF (median: 29.1 vs. 20.9 months, $\mathrm{P}=0.0025$ ), though event follow-up is yet to mature.

While this is the first head-to-head randomizedcontrolled trial demonstrating the superiority of GP to $\mathrm{PF}$, there are some caveats pertaining to this study. First of all, no stratification factor was employed during the randomization process. It has been previously shown that presence of liver metastasis is a poor prognostic group while lung metastasis is a favorable prognostic factor (11-13). Secondly, the usual dose of cisplatin is $100 \mathrm{mg} / \mathrm{m}^{2}$ given every 3 weeks when it is combined with $5-\mathrm{FU}$ in the recurrent/metastatic setting. However, it was set at $80 \mathrm{mg} / \mathrm{m}^{2}$ in both arms in the current study. It might be the intention of the authors to set it at this dose level as majority of their patients had been heavily exposed to platinum during their prior curative treatment. It is not known if this slightly diminished dose of cisplatin would carry any impact on tumor response and survival outcomes. Though induction chemotherapy was allowed in this study, only agents including platinum, 5-FU, docetaxel and paclitaxel but not gemcitabine were permitted in this setting. The study results may have become insignificant if gemcitabine is also allowed in the induction setting, as it is getting popularity as shown in recent studies (14-16). The current drug of choice may be further complicated by the recently published phase III RCT comparing induction chemotherapy with docetaxel, cisplatin and 5-FU (TPF) followed by concurrent chemoradiotherapy versus concurrent chemoradiotherapy (17). The more exposure to $5-\mathrm{FU}$ in the induction setting may diminish the efficacy when used in recurrent/metastatic diseases.

While chemotherapy is still the mainstay of treatment for incurable recurrent or metastatic NPC, novel systemic therapies have also been evaluated for the past decade. Various targeted therapeutic agents (including cetuximab, nimotuzumab, gefitinib, erlotinib, bevacizumab, sorafenib, sunitinib, pazopanib, axitinib) have been investigated alone or in combination with other chemotherapy drugs. However, the results were not impressive, no significant superiority in efficacy have been demonstrated.

Finally immunotherapy with immune checkpoint inhibitors has gradually emerged as a promising treatment modality for head and neck squamous cell carcinoma (HNSCC). Pembrolizumab in Keynote-012 phase Ib study, was shown to produce an objective response rate of $18 \%$, a median PFS of 2 months and a median OS of 13 months in the intention-to-treat population (18). This promising result has led to accelerated approval by Food and Drug Administration of the United States in August this year for the treatment of patients with recurrent or metastatic HNSCC with disease progression on or after platinumcontaining chemotherapy. More recently, nivolumab in the multi-center phase III randomized-controlled trial was superior to standard single-agent systemic therapy (methotrexate, docetaxel or cetuximab) (19). OS as the primary study endpoint was reached $(7.5 \mathrm{vs}$. 5.1 months, $\mathrm{P}=0.01)$. Though median PFS was similar between the two arms ( 2.0 vs. 2.3 months, $\mathrm{P}=0.32)$, the rate of PFS at 6 months was higher with nivolumab (19.7\% vs. 9.9\%). The objective response rate was also more promising $(13.3 \%$ vs. $5.8 \%)$. Perhaps it is prime time for these immunotherapeutic agents to be tested in NPC. The first phase I study on NPC demonstrated an 
objective response in $22.2 \%$ and disease control in $77.8 \%$ of a total of 44 patients (20). A least three phase II trials are ongoing to investigate their safety and efficacy in the recurrent/metastatic setting as second-line treatment or beyond after prior failure to platinum-based chemotherapy (NCT02339558, NCT02611960 and NCT02605967). Hopefully they will provide a new paradigm of treatment which can be further extended in the $1^{\text {st }}$ line setting.

In summary, gemcitabine and platinum has become the standard first-line treatment for recurrent/metastatic NPC. Future research focus should be directed to investigations of novel systemic therapies which can supplement or replace chemotherapy for better survival outcomes and more favorable toxicities.

\section{Acknowledgements}

None.

\section{Footnote}

Conflicts of Interest: The author has no conflicts of interest to declare.

\section{References}

1. Blanchard P, Lee A, Marguet S, et al. Chemotherapy and radiotherapy in nasopharyngeal carcinoma: an update of the MAC-NPC meta-analysis. Lancet Oncol 2015;16:645-55.

2. Lee AW, Sze WM, Au JS, et al. Treatment results for nasopharyngeal carcinoma in the modern era: the Hong Kong experience. Int J Radiat Oncol Biol Phys 2005;61:1107-16.

3. Wang TL, Tan YO. Cisplatin and 5-fluorouracil continuous infusion for metastatic nasopharyngeal carcinoma. Ann Acad Med Singapore 1991;20:601-3.

4. Au E, Ang PT. A phase II trial of 5-fluorouracil and cisplatinum in recurrent or metastatic nasopharyngeal carcinoma. Ann Oncol 1994;5:87-9.

5. Chi KH, Chan WK, Cooper DL, et al. A phase II study of outpatient chemotherapy with cisplatin, 5-fluorouracil, and leucovorin in nasopharyngeal carcinoma. Cancer 1994;73:247-52.

6. Lokich J, Anderson N. Carboplatin versus cisplatin in solid tumors: an analysis of the literature. Ann Oncol 1998;9:13-21.

7. Forastiere AA, Metch B, Schuller DE, et al. Randomized comparison of cisplatin plus fluorouracil and carboplatin plus fluorouracil versus methotrexate in advanced squamous-cell carcinoma of the head and neck: a Southwest Oncology Group study. J Clin Oncol 1992;10:1245-51.

8. De Andrés L, Brunet J, López-Pousa A, et al. Randomized trial of neoadjuvant cisplatin and fluorouracil versus carboplatin and fluorouracil in patients with stage IV-M0 head and neck cancer. J Clin Oncol 1995;13:1493-500.

9. Kua VF, Ismail F, Chee Ee Phua V, et al. Carboplatin/5fluorouracil as an alternative to cisplatin/5- fluorouracil for metastatic and recurrent head and neck squamous cell carcinoma and nasopharyngeal carcinoma. Asian Pac J Cancer Prev 2013;14:1121-6.

10. Zhang L, Huang Y, Hong S, et al. Gemcitabine plus cisplatin versus fluorouracil plus cisplatin in recurrent or metastatic nasopharyngeal carcinoma: a multicentre, randomised, open-label, phase 3 trial. Lancet 2016;388:1883-92.

11. Hui EP, Leung SF, Au JS, et al. Lung metastasis alone in nasopharyngeal carcinoma: a relatively favorable prognostic group. A study by the Hong Kong Nasopharyngeal Carcinoma Study Group. Cancer 2004;101:300-6.

12. Winter H, Meimarakis G, Hoffmann G, et al. Does surgical resection of pulmonary metastases of head and neck cancer improve survival? Ann Surg Oncol 2008;15:2915-26.

13. Ong YK, Heng DM, Chung B, et al. Design of a prognostic index score for metastatic nasopharyngeal carcinoma. Eur J Cancer 2003;39:1535-41.

14. Tan T, Lim WT, Fong KW, et al. Concurrent chemoradiation with or without induction gemcitabine, Carboplatin, and Paclitaxel: a randomized, phase 2/3 trial in locally advanced nasopharyngeal carcinoma. Int J Radiat Oncol Biol Phys 2015;91:952-60.

15. Lim AM, Corry J, Collins M, et al. A phase II study of induction carboplatin and gemcitabine followed by chemoradiotherapy for the treatment of locally advanced nasopharyngeal carcinoma. Oral Oncol 2013;49:468-74.

16. Yau TK, Lee AW, Wong DH, et al. Treatment of Stage IV(A-B) nasopharyngeal carcinoma by inductionconcurrent chemoradiotherapy and accelerated fractionation: impact of chemotherapy schemes. Int J Radiat Oncol Biol Phys 2006;66:1004-10.

17. Sun Y, Li WF, Chen NY, et al. Induction chemotherapy plus concurrent chemoradiotherapy versus concurrent chemoradiotherapy alone in locoregionally advanced nasopharyngeal carcinoma: a phase 3 , multicentre, 
randomised controlled trial. Lancet Oncol 2016;17:1509-20.

18. Seiwert TY, Burtness B, Mehra R, et al. Safety and clinical activity of pembrolizumab for treatment of recurrent or metastatic squamous cell carcinoma of the head and neck (KEYNOTE-012): an open-label, multicentre, phase $1 \mathrm{~b}$ trial. Lancet Oncol 2016;17:956-65.

Cite this article as: Lee V. How far have we achieved by systemic therapy for recurrent/metastatic nasopharyngeal carcinoma? Transl Cancer Res 2016;5(Suppl 7):S1424-S1427. doi: 10.21037/tcr.2016.12.25
19. Ferris RL, Blumenschein G Jr, Fayette J, et al. Nivolumab for Recurrent Squamous-Cell Carcinoma of the Head and Neck. N Engl J Med 2016;375:1856-67.

20. Hsu C, Lee SH, Ejadi S, et al. Antitumor activity and safety of pembrolizumab in patients with PD-L1-positive nasopharyngeal carcinoma: Interim results from a phase $1 \mathrm{~b}$ study. Ann Oncol 2015;51:ix94. 